\title{
Bio-inspired dental fillings
}

\author{
Hans Deyhle ${ }^{\mathrm{a}, \mathrm{b}}$, Oliver Bunk ${ }^{\mathrm{c}}$, Stefan Buser ${ }^{\mathrm{a}, \mathrm{b}}$, Gabriel Krastl ${ }^{\mathrm{a}}$, Nicola U. Zitzmann ${ }^{\mathrm{a}}$, \\ Bernd Ilgenstein $^{\mathrm{a}}$, Felix Beckmann ${ }^{\mathrm{d}}$, Franz Pfeiffer ${ }^{\mathrm{c}, \mathrm{e}}$, Roland Weiger ${ }^{\mathrm{a}}$, and Bert Müller*a, \\ ${ }^{a}$ School of Dental Medicine, University of Basel, Hebelstrasse 3, 4056 Basel, Switzerland; \\ ${ }^{\mathrm{b}}$ Biomaterials Science Center, University of Basel, c/o University Hospital, 4031 Basel, Switzerland; \\ ${ }^{c}$ Paul Scherrer Institute, 5232 Villigen-PSI, Switzerland; \\ ${ }^{\mathrm{d}}$ GKSS Research Center, Max-Planck-Str. 1, 21502 Geesthacht, Germany; \\ ${ }^{e}$ Department of Physics (E17), Technical University Munich, 85748 Garching, Germany
}

\begin{abstract}
Human teeth are anisotropic composites. Dentin as the core material of the tooth consists of nanometer-sized calcium phosphate crystallites embedded in collagen fiber networks. It shows its anisotropy on the micrometer scale by its welloriented microtubules. The detailed three-dimensional nanostructure of the hard tissues namely dentin and enamel, however, is not understood, although numerous studies on the anisotropic mechanical properties have been performed and evaluated to explain the tooth function including the enamel-dentin junction acting as effective crack barrier. Small angle X-ray scattering (SAXS) with a spatial resolution in the $10 \mu \mathrm{m}$ range allows determining the size and orientation of the constituents on the nanometer scale with reasonable precision. So far, only some dental materials, i.e. the fiber reinforced posts exhibit anisotropic properties related to the micrometer-size glass fibers. Dental fillings, composed of nanostructures oriented similar to the natural hard tissues of teeth, however, do not exist at all. The current X-ray-based investigations of extracted human teeth provide evidence for oriented micro- and nanostructures in dentin and enamel. These fundamental quantitative findings result in profound knowledge to develop biologically inspired dental fillings with superior resistance to thermal and mechanical shocks.
\end{abstract}

Keywords: human tooth, dentin, enamel, small angle X-ray scattering, micro computed tomography, microtubules, anisotropy, microstructure, nanometer scale, synchrotron radiation

\section{INTRODUCTION}

Enamel and dentin of human teeth belong to the anisotropic biological materials. Enamel is known as highly mineralized hard and brittle substance [1]. On the micrometer scale, oriented enamel rods or prisms can be observed, which are the result of polarized columnar cells termed ameloblasts. These enamel rods mainly consist of densely packed calcium phosphates. It is also found within the spaces between the crystallites. The only difference between the calcium phosphate in and between the rods is their orientation on the nanometer scale.

Dentin can be regarded as a natural composite consisting of carbonate-rich calcium phosphate nanometer-sized crystallites embedded in collagen fiber networks. As the result of the different composition and density X-ray-based imaging easily permits the discrimination between the two hard tissues dentin and enamel.

While the principal anatomy of the tooth has been understood since decades, the detailed three-dimensional micro- and nanostructure especially at the dentin-enamel junction is still matter of research (see, e.g. [1-3]). Electron microscopy micrographs provide impressive qualitative insights into the crystallite morphology, but the quantitative evaluation is time-consuming and complex as well as distorted by the sectioning artifacts. Nevertheless, there are several successful attempts to uncover the size, orientation, and density of the apatite crystallites as well as the organization of the collagen fibrils in the three-dimensional space.

*bert.mueller@unibas.ch; phone 4161 265-9660; fax 4161 265-9699; www.bmc.unibas.ch

Biomimetics and Bioinspiration, edited by Raúl J. Martín-Palma, Akhlesh Lakhtakia, Proc. of SPIE Vol. 7401, 74010E · @ 2009 SPIE · CCC code: 0277-786X/09/\$18 · doi: 10.1117/12.827437 
The studies include synchrotron radiation-based small angle X-ray scattering (SAXS) with a spatial resolution down to $200 \mu \mathrm{m}$ [3]. Although these SAXS studies already indicate local variations in the ordered phases within the dentin, the restricted spatial resolution has not allowed any conclusion about the enamel-dentin junction that is of paramount importance for the mechanical properties of the entire teeth [1]. The present investigation evaluates how far scanning SAXS with a spatial resolution in the range of $10 \mu \mathrm{m}$ [4] can contribute to an improved understanding of the nanoarchitecture of dentin and enamel in particular near the dentin-enamel junction, where the ordered dentinal tubules are supposed to end. This morphological transition adapts the mechanical properties between the enamel of high strength and brittleness and the supportive compliant dentin [1]. So far, dental fillings used to repair (caries-induced) defects do not exhibit any similar micro- or nano-architecture neither anisotropic mechanical properties as observed for the two biological materials. In this context, the current study on the three-dimensional micro- and nano-architecture of the underlying structural elements should enable the realization of bio-mimetic dental fillings with superior resistance to thermal and, more important, to mechanical shocks.

\section{MATERIALS AND METHODS}

\subsection{Tooth selection and preparation for X-ray imaging}

An extracted non-restored molar tooth of the fourth quadrant without any visible damage was selected for the experiments. The human tooth was caries-free as it did not show any clinical sign of enamel caries. For the measurements the extracted tooth was placed into an Eppendorf tube to avoid dehydration.

\subsection{Conventional micro computed tomography of teeth}

Micro computed tomography $(\mu \mathrm{CT})$ measurements of the complete tooth in the liquid-filled Eppendorf tube were performed with the system $1174^{\mathrm{TM}}$ (SkyScan, Kontich, Belgium) $[5,6]$. The fixed specimen rotation stage allows for positioning in one direction perpendicular to the X-ray beam. The X-ray absorption of the tooth required the highest possible accelerating voltage of $50 \mathrm{kV}$ and the application of a $0.5 \mathrm{~mm}$-thick aluminum filter. The focus between the Xray scintillator and the one megapixel $\mathrm{CCD}$ detector was adjusted to obtain an isotropic pixel size of $28.8 \mu \mathrm{m}$. 900 projections from 0 to $360^{\circ}$ in steps of $0.4^{\circ}$ were recorded using a beam current of $800 \mu \mathrm{A}$. The reconstruction was performed with a modified Feldkamp algorithm using SkyScan Nrecon ${ }^{\mathrm{TM}}$ software.
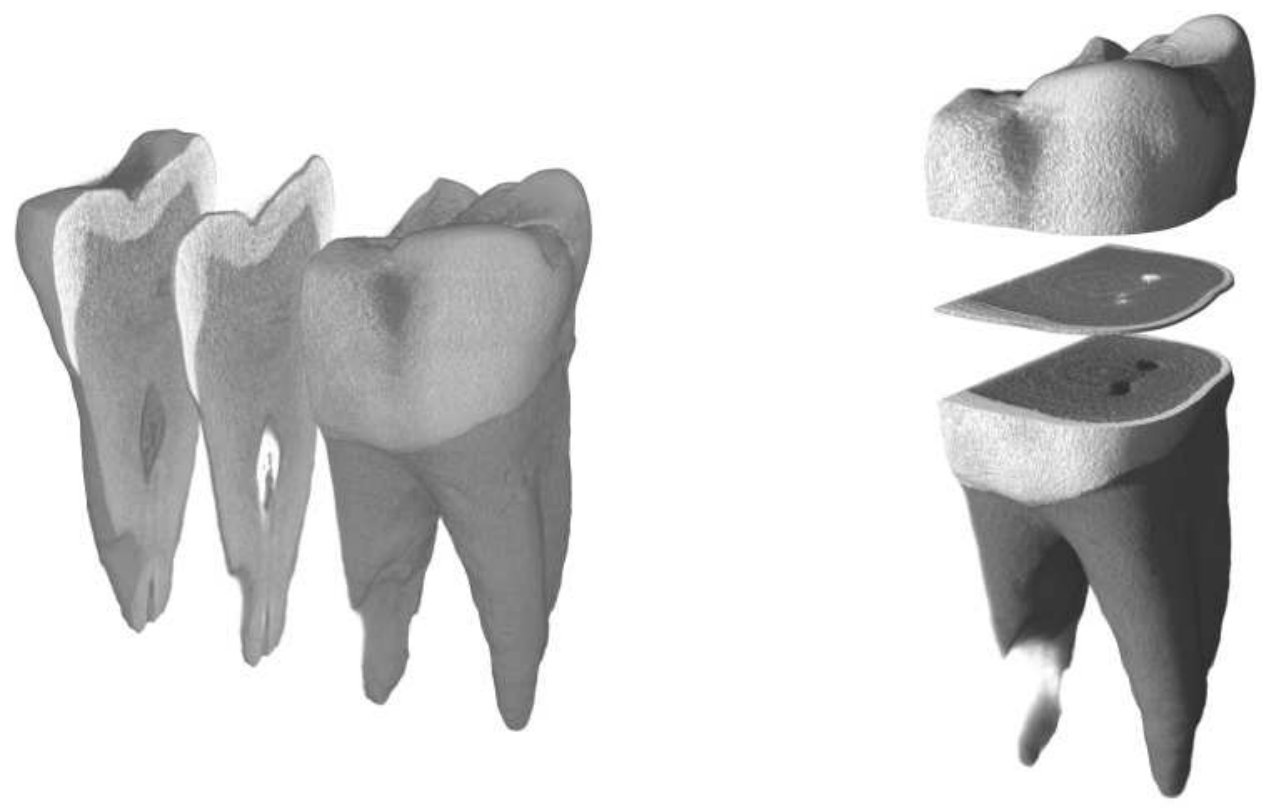

Fig. 1. Preparation of teeth slices for the high-resolution measurements parallel and perpendicular to the tooth axis. The images were generated from the $\mu \mathrm{CT}$-data using the software VG Studio Max 1.2.1 (Volume Graphics, Heidelberg, Germany). 


\subsection{Specimen preparation}

Based on the $\mu \mathrm{CT}$ results of the complete tooth, Figure 1 schematically shows the harvesting of slices with thicknesses of about $200 \mu \mathrm{m}$ and $300 \mu \mathrm{m}$, respectively. The slices were obtained parallel and perpendicular to the tooth axis. A saw (Exact Apparatebau GmbH, Norderstedt, Germany) equipped with a $0.2 \mathrm{~mm}$ cutting diamond band served for the slice preparation.

\subsection{Synchrotron radiation-based micro computed tomography}

The SR $\mu$ CT-measurements were performed at two different beamlines: W 2 (HASYLAB at DESY, Hamburg, Germany) and TOMCAT (SLS at PSI, Villigen, Switzerland).

The beamline W 2 with its standard setup for absorption contrast tomography [7], operated by the GKSS Research Center, served for the simultaneous visualization of a series of teeth slices combined in one container filled with phosphate buffer saline (PBS). The photon energy was set to $45 \mathrm{keV}$. The present study included two datasets at different heights, which were combined after reconstruction with pixel resolution. The rotation axis was chosen asymmetrical to the incoming X-ray beam to increase the spatial resolution [8]. Therefore, the complementary projections were combined before reconstruction. The number of projections corresponded to 1441 acquired by rotating the sample in steps of $0.25^{\circ}$ from 0 to $360^{\circ}$. The filtered back-projection algorithm served for the slice-wise reconstruction [9]. In order to improve the density resolution at the restricted number of projections, the data, recorded with a pixel size of $4.6 \mu \mathrm{m}$, were binned by a factor of two before reconstruction [10]. The spatial resolution determined from the $10 \%$ value of the modulated transfer function of a highly X-ray absorbing edge corresponded to $8.8 \mu \mathrm{m}[11]$.

The SR $\mu$ CT-measurements at TOMCAT [12] were carried out in absorption contrast mode using a photon energy of $15 \mathrm{keV}$ with a band width of $2 \%$ to $3 \%$ and an exposure time of $170 \mathrm{~ms}$ per projection. The absorption contrast contains edge enhancement as well. For these experiments a rod was cut out of the tooth slice so that the specimen presented with a maximum diameter of $0.7 \mathrm{~mm}$. This setting permitted high-resolution measurements with a pixel size of $0.37 \mu \mathrm{m}$. The specimen held in air was rotated in steps of $0.12^{\circ}$ between $0^{\circ}$ and $180^{\circ}$ to record the projections. Reconstructed data were obtained by means of the filtered back-projection available at the beamline, where 9 height steps were acquired to follow a reasonable volume within dentin and enamel as well as of the dentin-enamel junction.

\subsection{Small angle $X$-ray scattering}

Small angle X-ray scattering (SAXS) is a powerful method that profits from the elastic scattering of X-rays at features within the nanometer range [13]. SAXS is usually restricted to scattering angles well below $10^{\circ}$. SAXS data contain quantitative information averaged on the illuminated area.

As schematically shown in Figure 2, the X-ray beam with the selected photon energy is scattered by the nanometer-sized features of the tooth slice. The interaction of the $\mathrm{keV}$-photons with matter, however, is rather weak, so that most of the beam simply penetrates the tooth slice. In order to detect the weak scattered intensities, a beam stop is usually incorporated (see Figure 2).

The SAXS measurements were performed at the cSAXS beamline of the Swiss Light Source (Paul Scherrer Institut, Villigen, Switzerland). A monochromatic X-ray beam with a photon energy of $18.58 \mathrm{keV}$ was focused to a spot of about $20 \mu \mathrm{m} \times 5 \mu \mathrm{m}$ for the raster scan measurements. Two modules of a PILATUS detector [14] with about 190 '000 pixels in total have been used for the detection. To speed up the data acquisition, the tooth slice was moving at constant speed along a line of the $2 \mathrm{D}$ raster pattern, while the detector was continuously recording data. The detector was operated with $20 \mathrm{~ms}$ exposure time and $8 \mathrm{~ms}$ readout time. This selection corresponds to a frame rate of $36 \mathrm{~Hz}$.

In order to determine information on the abundance of nanostructures of interest within the illuminated point of the raster scan, the recorded intensity was averaged within the related ring around the through beam. The orientation and the anisotropy of these nanostructures follow the angular intensity distribution along the ring [4]. One finally obtains an intensity map with the color-coded orientation for the selected nanometer range. 


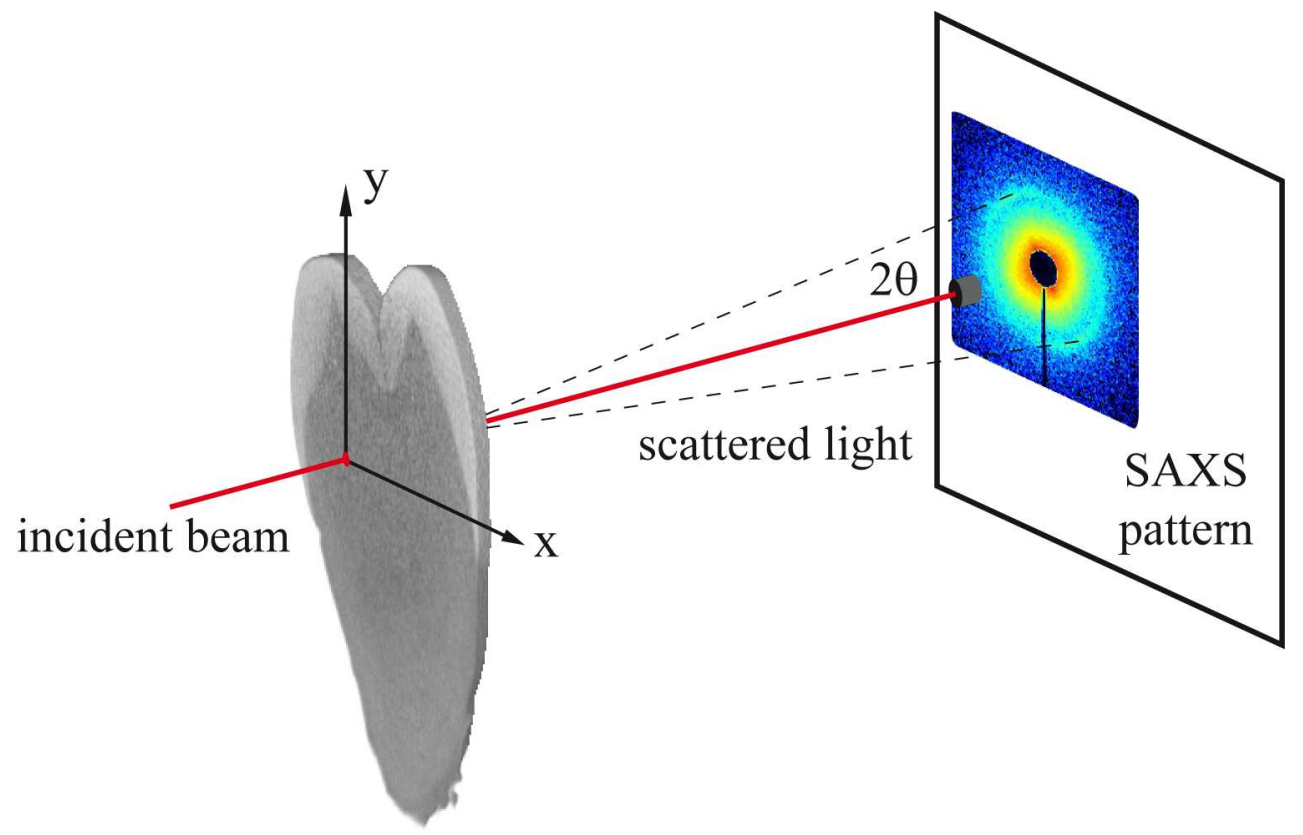

Fig. 2. The X-ray beam, about $5 \mu \mathrm{m} \times 20 \mu \mathrm{m}$ wide, perpendicularly hits the tooth slice that can be moved along $\mathrm{x}$ and $\mathrm{y}$ directions. For each (x,y)-position a SAXS pattern is recorded. Finally a ring of each SAXS pattern around the direct beam is analyzed to determine the size and orientation of the nanometer-sized features of dentin and enamel.

\section{RESULTS}

\subsection{Micro computed tomography to characterize the tooth slices}

Synchrotron radiation-based micro computed tomography (SR $\mu \mathrm{CT}$ ) served for both the determination of the outer shape of the entire slice, which includes the measurement of the slice thickness, and the internal microstructure of the tooth slice.

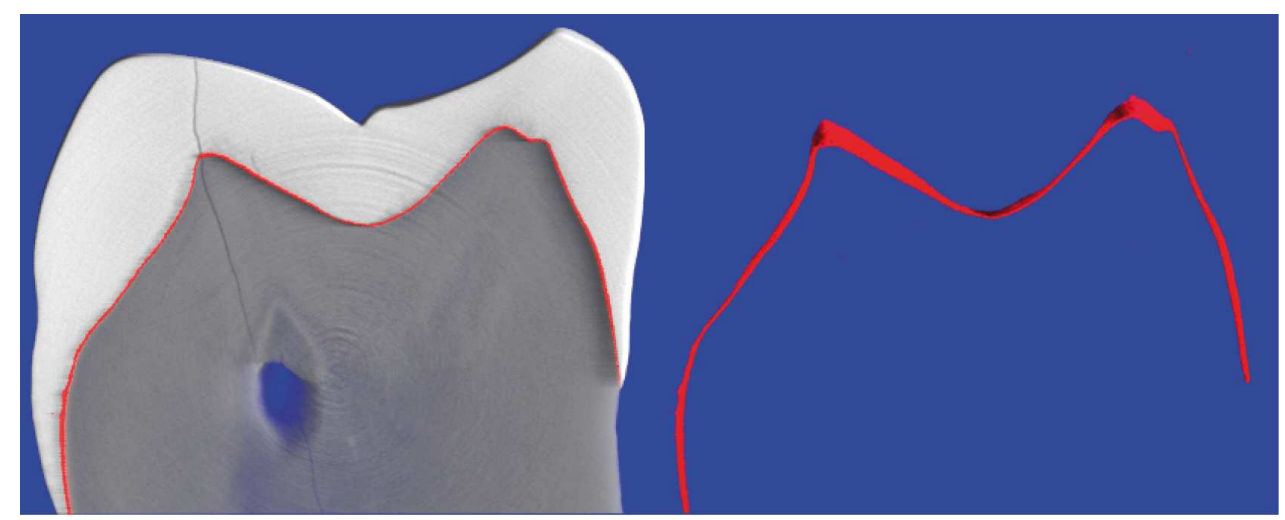

Fig. 3. The high-resolution tomography experiments of the tooth slice enable the segmentation of two homogeneous components (enamel and dentin) and the interface (as the result of the partial volume effect). In addition, the image also shows a crack and an abrasion on the enamel.

One can perfectly discriminate between enamel and dentin because of the different X-ray absorption (see Figure 3). Dentin and enamel, however, appear as homogeneous materials. Hence, the value of the SR $\mu$ CT remains in the measurement of the slice thickness and parallelism and, more important, in the local orientation of the dental-enamel junction. If the dental-enamel junction is parallel to the beam direction for the SAXS measurements, one can draw 
conclusions about this interface. If the interface, however, shows an angle of $45^{\circ}$ for instance, the SAXS-measurements yields a mean value of both enamel and dentin.

\subsection{Microstructure of dentin: Visualization of tubules}

For the high-resolution tomography, a rod was cut from one $500 \mu \mathrm{m}$ thick tooth slice. This rod fits well into a cylinder less than $0.7 \mathrm{~mm}$ in diameter and, therefore, allows for sub-micrometer resolution tomography. Figure 3 represents a series of nine slices perpendicular to the rod axis, which are $74 \mu \mathrm{m}$ apart from each other. The enamel given in light gray in the first two slices exhibits a stronger X-ray absorption than the dentin. Thus, enamel and dentin can be easily segmented. In these high-resolution edge-enhanced images the enamel does not exhibit a homogeneous X-ray absorption as in the X-ray imaging presented above and well known from conventional X-ray imaging techniques but certain variations in composition that includes very few tubular microstructures.
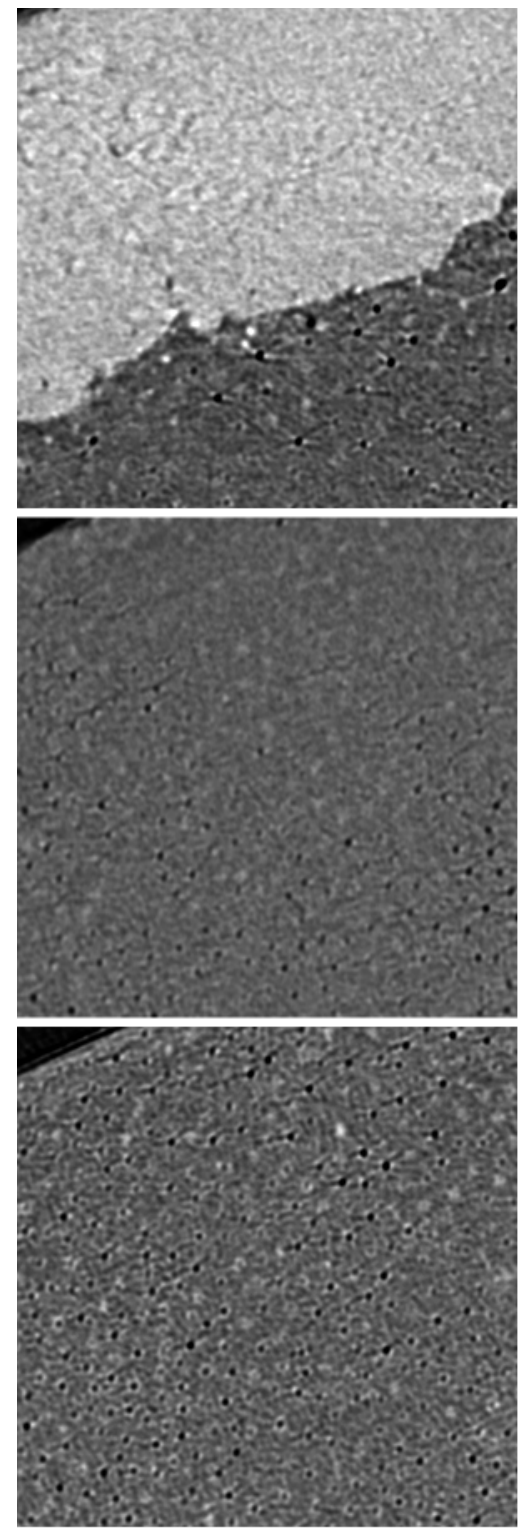
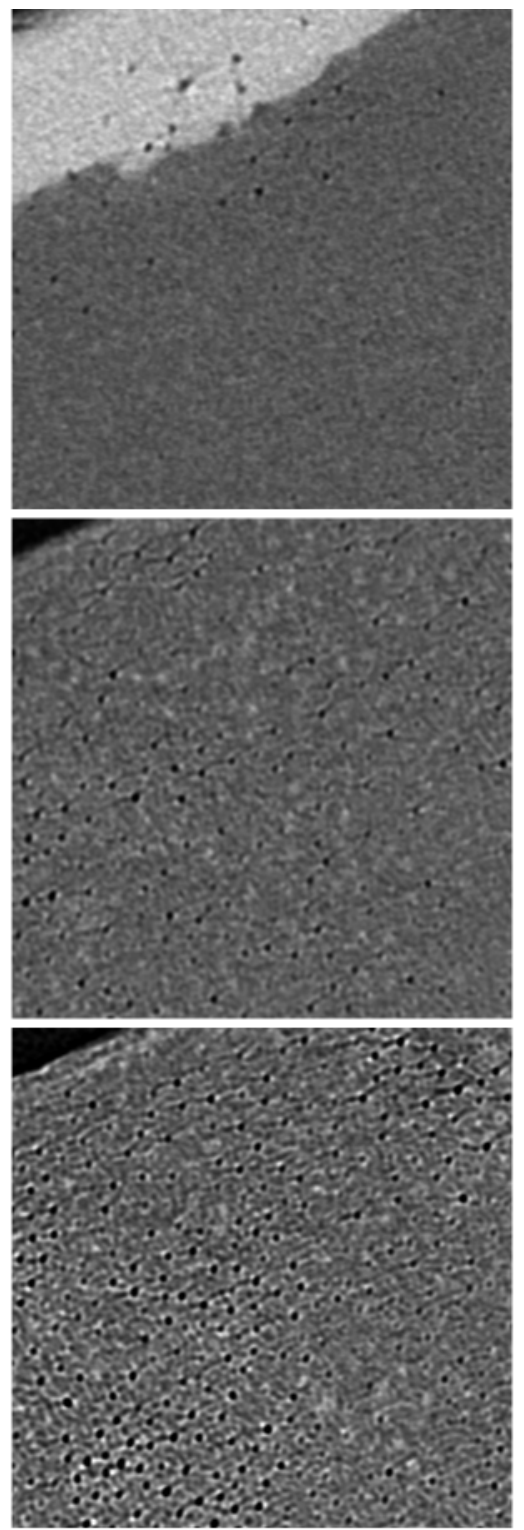
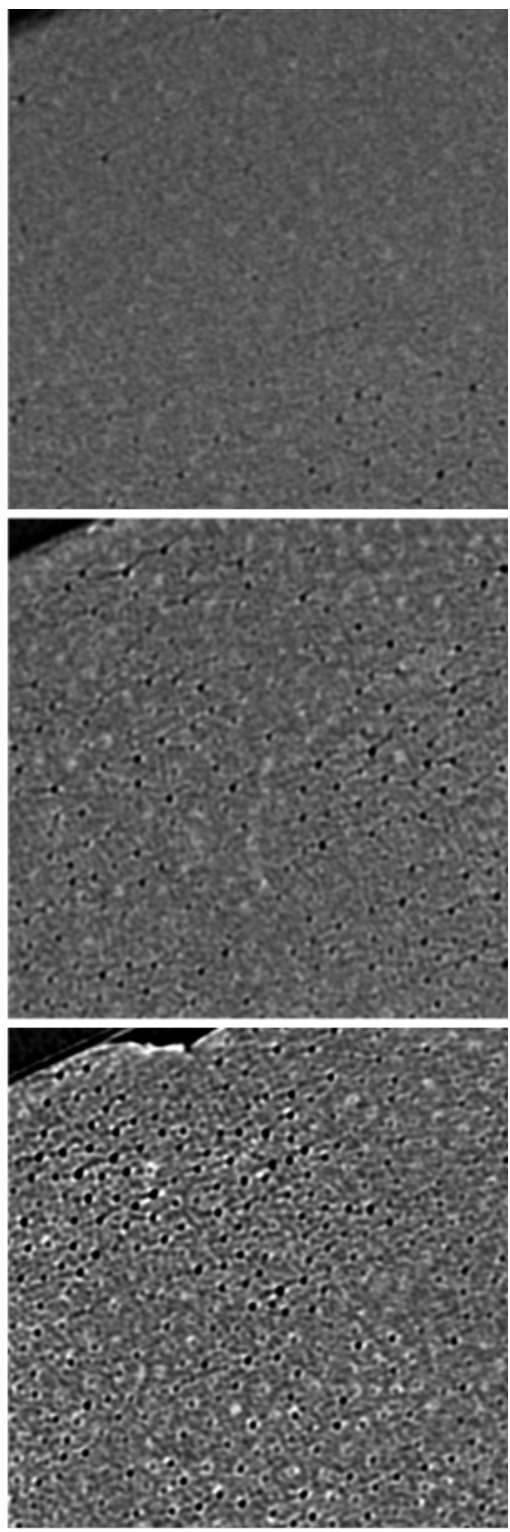

$50 \mu \mathrm{m}$

Fig. 4. The series of slices shows the density distribution of the tubules, which becomes smaller and smaller towards the dentin-enamel junction. The distance between the slices corresponds to $74 \mu \mathrm{m}$ each. 
Within the dentin structure thousands of tubules are visible. The density of these tubules, however, varies and depends on the distance to the dentin-enamel junction. In general, the tubule density decreases towards the interface to the enamel, as indicated in Figures 4 and 5.

Figure 5 shows the density distribution of the tubules, which are oriented in a parallel manner. Here, the distance between the slices also corresponds to $74 \mu \mathrm{m}$. It is also recognized in Figure 5 that although the tubules are usually parallel, their orientation can change to become perpendicular to the dentin-enamel junction.

The tubules do not appear as simple cavities, which would become visible as very thin black cylinders. Almost each tubule contains a bright halo with a thickness of one or two micrometers.
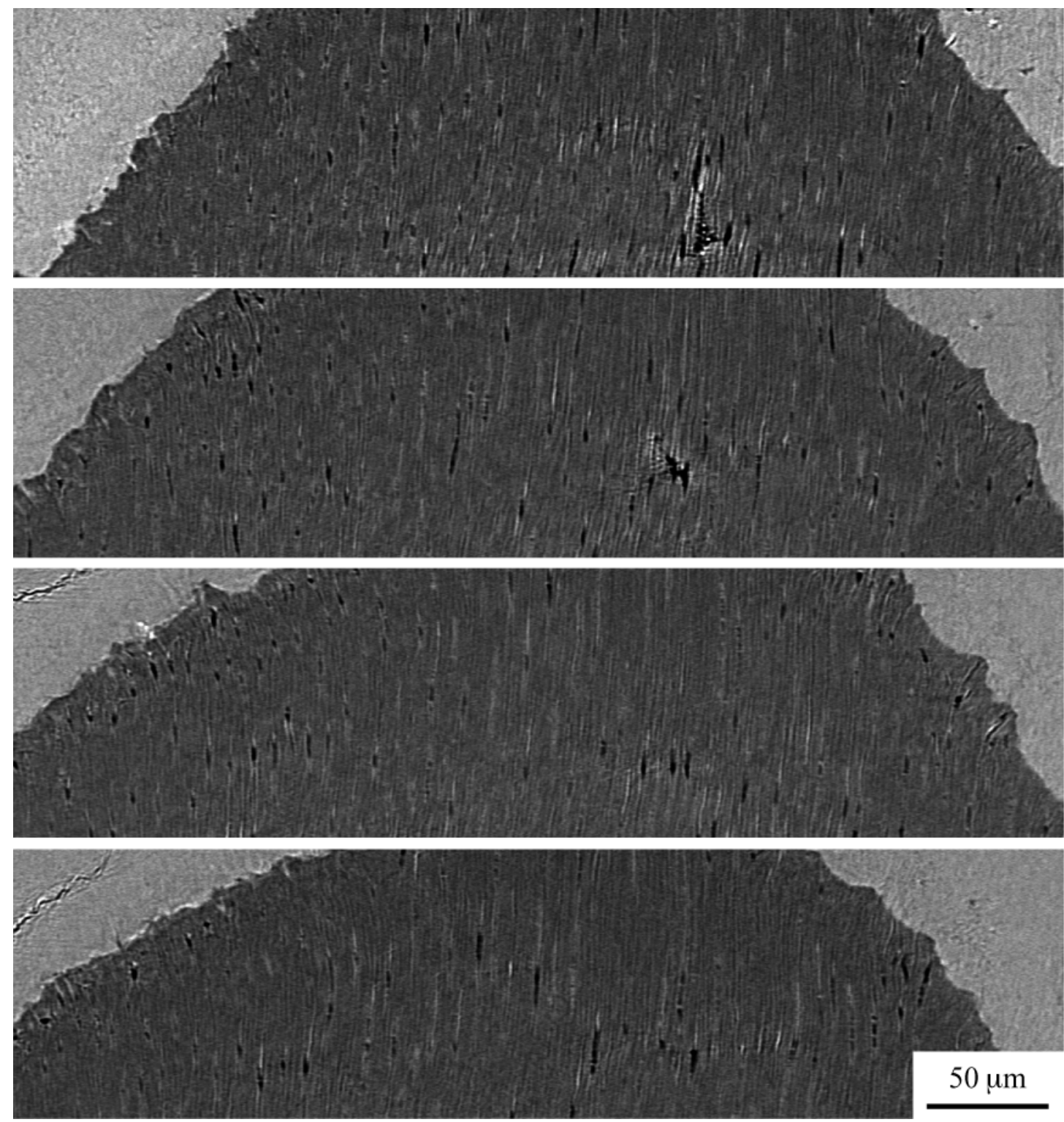

Fig. 5. The series of selected slices parallel to the tooth axis demonstrates the parallel orientation of the tubules within the dentin. Their density is reduced towards the dentin-enamel junction. The crack in the enamel is attributed to a preparation artifact. 


\subsection{Nanostructure of enamel and dentin}

The SAXS measurements prove the preferential orientation of the nanostructures present in dentin and enamel. Figure 6 shows the cut perpendicular to the tooth axis. The nanostructures in the dentin are hardly ordered as indicated by the gray color. Only in the $20 \mathrm{~nm}$ range the nanostructures are oriented towards the growth direction. The light color, however, indicates that the number of scatters is rather weak. For the $20 \mathrm{~nm}$ range, however, one can differentiate between two different kinds of dentin. The inner part of the tooth contains more scatter centers, which are less ordered, whereas the part towards the enamel exhibit better ordered nanostructures of rather small density.
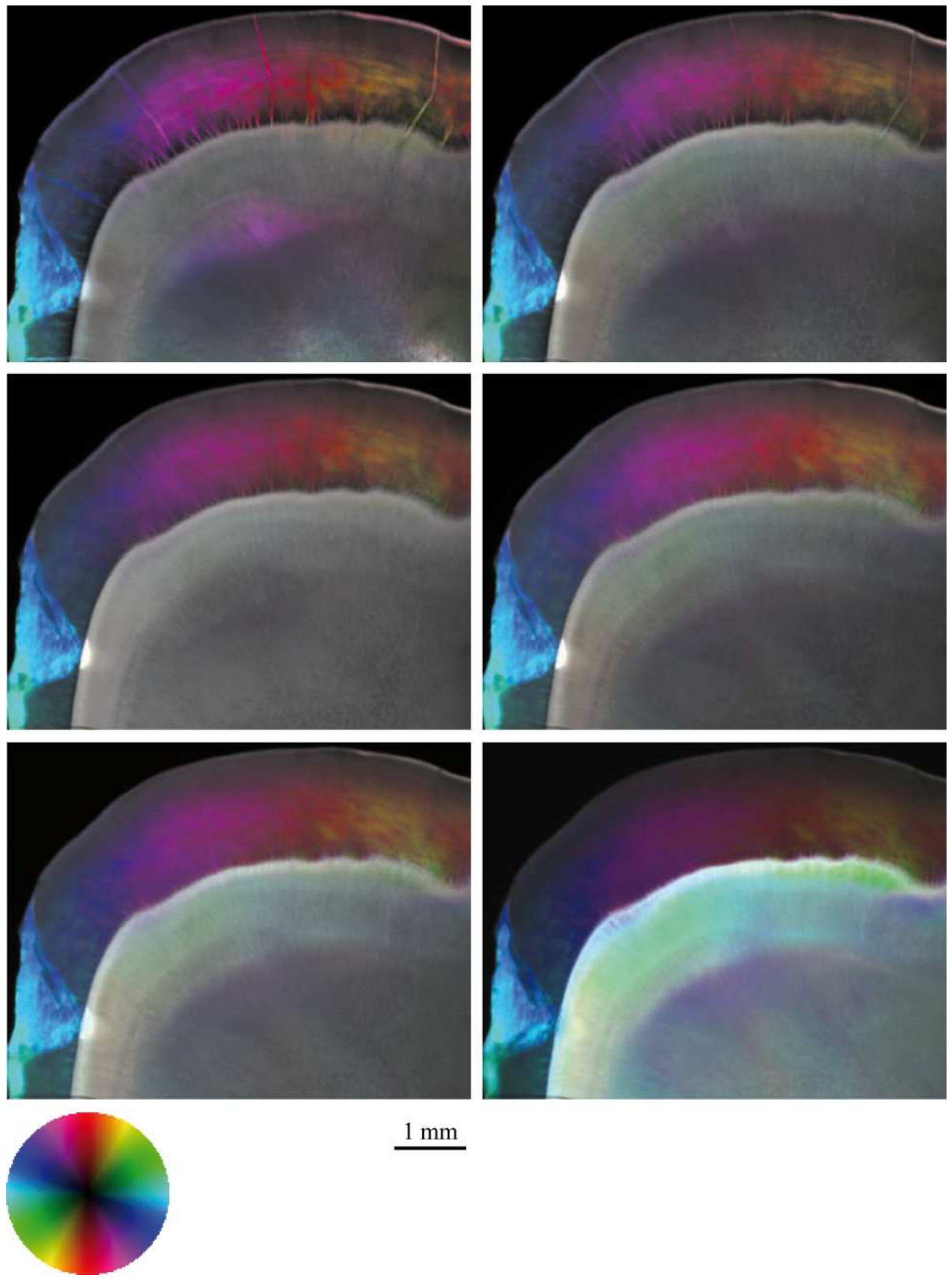

Fig. 6. The representation of the SAXS measurement demonstrates that nanostructures within dentin and enamel are often oriented from the center of the tooth towards the periphery. The images are obtained for the different length scales: 14 to $24 \mathrm{~nm}$ (top, left), 24 to $39 \mathrm{~nm}, 40$ to $52 \mathrm{~nm}, 53$ to $71 \mathrm{~nm}, 73$ to $173 \mathrm{~nm}$ and 185 to $231 \mathrm{~nm}$ (bottom, right). The colored circular area denotes the orientation of the scattered intensity. 
The enamel contains much more nanometer-sized features than the dentin in the range between 10 and $200 \mathrm{~nm}$. They are clearly oriented in the central part, best seen within the $200 \mathrm{~nm}$ range. Although, there exist close similarities between the different nanometer scales, only the larger nanostructures show prominent features that are even crack-like traveling through the whole enamel structure of the tooth. The turquoise colored part on the left side of the images belongs to the characteristics of this particular slice. This part is significantly thinner, because a part of the enamel was broken off during the preparation of the slice.
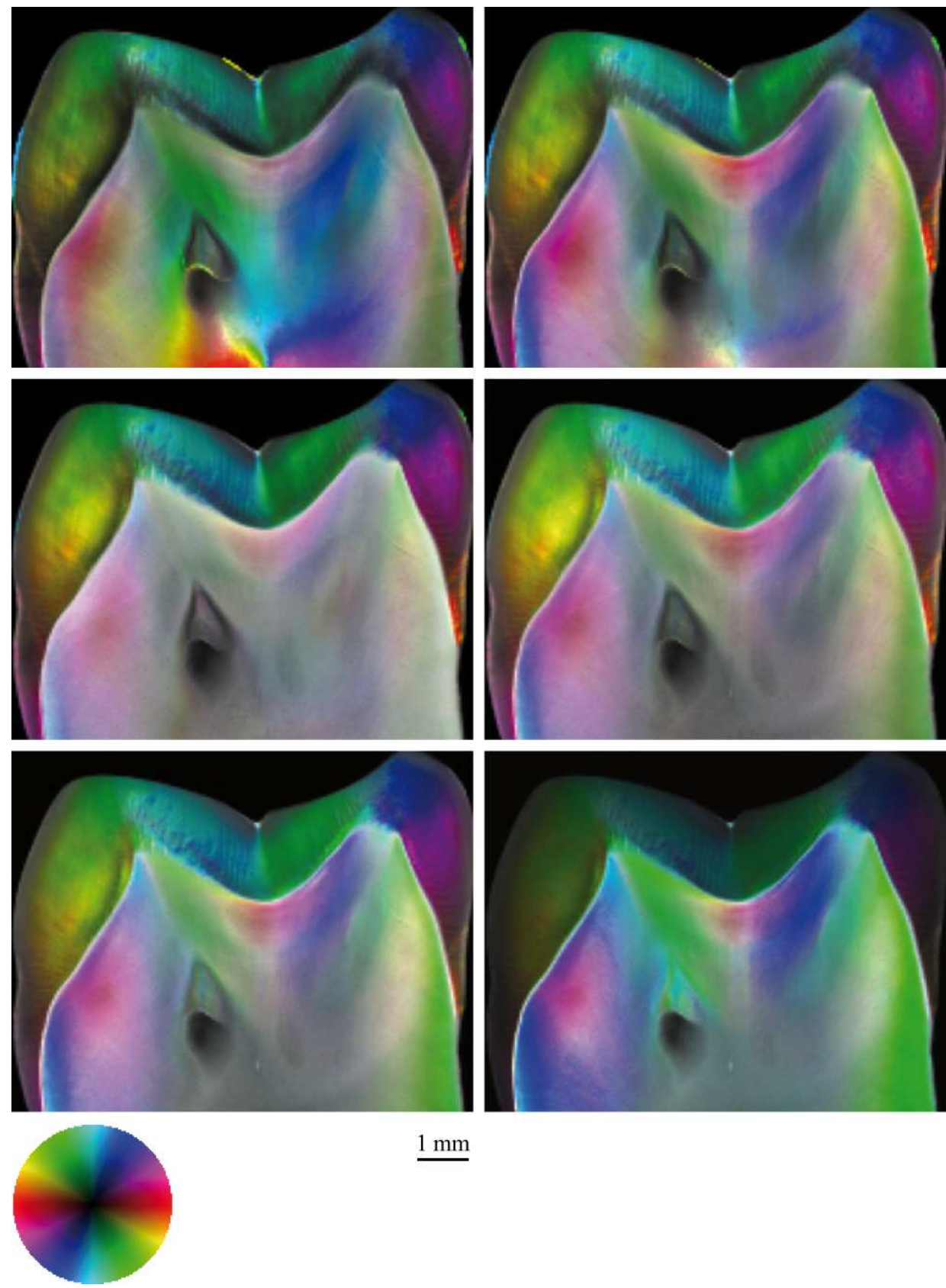

$\underline{1 \mathrm{~mm}}$

Fig. 7. The representation of the SAXS data parallel to the tooth axis reveals a preferential orientation of features in the range between 10 and $200 \mathrm{~nm}$ for both enamel and dentin. The orientation, however, is significantly different for both hard tissue components. The images are obtained for the different length scales: 14 to $24 \mathrm{~nm}$ (top, left), 24 to $39 \mathrm{~nm}, 40$ to $52 \mathrm{~nm}, 53$ to $71 \mathrm{~nm}, 73$ to $173 \mathrm{~nm}$ and 185 to $231 \mathrm{~nm}$ (bottom, right). 
The SAXS data of Figure 7 are obtained from a slice parallel to the tooth axis with a thickness of $(270 \pm 10) \mu \mathrm{m}$. In comparison to Figure 6 it demonstrates that the nanostructures of the hard tissues of the tooth are mainly oriented from top to bottom.

The SAXS experiments allow discriminating between enamel and dentin, since the nanostructures of the same frequencies are oriented in different directions. Nanostructures of the enamel that are below $24 \mathrm{~nm}$ do not exhibit the preferential orientation as observed among the larger ones. The color-coded images further uncover that the nanostructures within certain limits abruptly change their orientation at the dentin-enamel junction. Analyzing the $\mathrm{SR} \mu \mathrm{CT}$ data of the tooth slices, it is concluded that the transparent line on the left side of the tooth resulted from the interface between of dentin and enamel with rather irregular nanostructures. The abrasion on the left part of the tooth does not affect the orientation of the adjacent nanostructures.

\section{DISCUSSION AND CONCLUSIONS}

Conventional X-ray imaging techniques allow extracting the tooth geometry and the intensity-based segmentation [15] of dentin and enamel. The spatial and density resolution, however, are insufficient to uncover the micro- and the nanostructure of teeth tissues. The quantitative determination of the composition is especially demanding [16], since the dentin belongs to biological tissues with related diversification and property variations. Tomography with true micrometer resolution permits the visualization of the dentin tubules $[17,18]$. Contrary to electron microscopy the tomographic imaging allows visualizing the tubules in the three-dimensional space and not only at the surface. Hence, the density and orientation of the tubules are quantitatively accessible. The bright halo of the tubules can be attributed to the enhanced density around each tubule and/or to the edge enhancement as the result of the coherent X-ray beam. In the slices of Figure 5 some cracks within the enamel are recognized. Such cracks are probably the result of the imperfect preparation procedure.

The current investigations reveal strong anisotropic morphologies of dentin and enamel on the micro- and nanometer scale. The orientation of the tubules follows the growth direction that corresponds to the main direction of mechanical loading. The SAXS data, which uncover the nano-architecture of the hard tissues, also show preferential orientation towards the growth directions. Interestingly, the orientations of the nanostructures within the dentin and the enamel are altered. Within certain regions, the nanostructures are almost perpendicular to each other at the dentin-enamel junction.

This fundamental knowledge should be applied for the development of biologically inspired dental fillings, i.e. to realize man-made materials with a micro- and nano-architecture similar to enamel and dentin. If caries has significantly damaged the enamel and dentin of the tooth, the dentist removes the damaged part and re-builds it by means of conventional isotropic dental composite materials that do not resemble the anisotropic structure of human teeth. The use of such a material that tends to shrink is also the reason, why the dentist usually removes more enamel than really necessary. Overhangs, as represented in Figure 8, are generally avoided to prevent the potential cracking. Therefore, improved fillings are highly desirable to achieve better results by less invasive treatment modalities.

The challenge for the realization of the biologically inspired fillings lies in the arrangement of the nanometer-sized building blocks. At least two different layers, namely for the dentin and for the enamel have to be foreseen in order to achieve the different preferential orientation of the nanostructures.

One could imagine various approaches to imitate the hard tissues of the human teeth. For example, crystalline growth processes, which take place at non-equilibrium conditions with gradients in temperature or concentration, give rise to nanostructures with strong anisotropies [19]. Unfortunately such processes are difficult to be controlled and, hence, impractical for patient treatment. Furthermore, one could apply fiber composites as already successfully incorporated into dental posts. The appropriate orientation of the fibers, however, is demanding. Building blocks of charged or dipolar units such as dedicated carbon nano-tubes would result in parallel arrangements of the nanostructures. The dashed curves in Figure 8 represent the potential arrangements of charged nano-tubes. Because of the charge, the nano-tubes exhibit a repulsive interaction that leads to almost parallel equidistant alignments [20]. Such dental fillings should have superior resistance to thermal and mechanical shocks as known from the hardest substance in the human body - the enamel. 


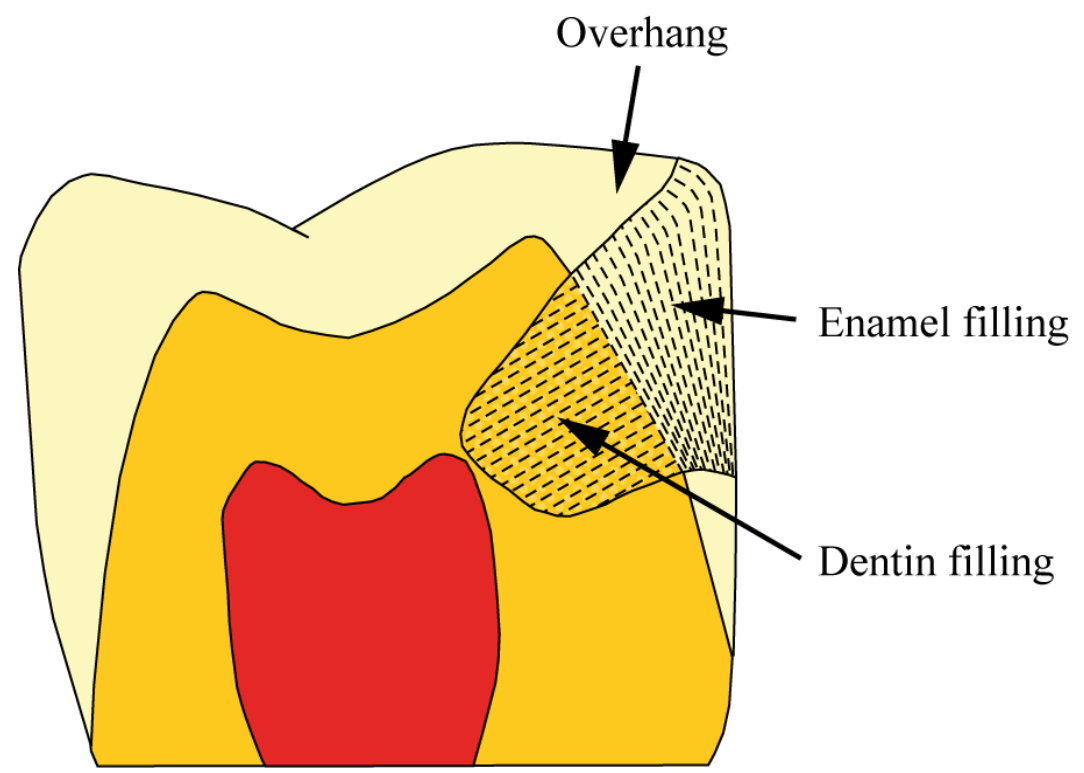

Fig. 8. The biomimetic and biologically inspired dental fillings should contain nanostructures such as charged carbon nanotubes that become aligned because of the repulsive interactions. Their size and orientation should be analogue to the undamaged dentin and enamel.

\section{ACKNOWLEDGEMENTS}

The beamtime at the synchrotron radiation facilities was kindly provided within the approved proposals I-20080224 EC (HASYLAB at DESY) and 20080959 (SLS at PSI). The technical support of F. Schmidli (Basel) for the tooth preparation and characterization is gratefully acknowledged.

\section{REFERENCES}

[1] V. Imbeni, J. J. Kruzic, G. W. Marshall et al., "The dentin-enamel junction and the fracture of human teeth," Nature Materials, 4, 229-232 (2005).

[2] M. Al-Jawad, A. Streuwer, S. H. Kilcoyne et al., "2D mapping of texture and lattice parameters of dental enamel," Biomaterials, 28, 2908-2914 (2007).

[3] J. H. Kinney, J. A. Pople, G. W. Marshall et al., "Collagen orientation and crystallite size in human dentin: a small angle X-ray scattering study," Calcif. Tissue Int., 69, 31-37 (2001).

[4] O. Bunk, M. Bech, T. H. Jensen et al., "Multimodal X-ray scatter imaging," submitted, (2009).

[5] S. Drews, F. Beckmann, J. Herzen et al., [Comparative micro computed tomography study of a vertebral body] SPIE, San Diego (2008).

[6] A. Papadimitropoulos, S. Friess, F. Beckmann et al., [Comparative study of desktop- and synchrotron radiationbased micro computed tomography analyzing cell-seeded scaffolds in tissue engineering of bone] SPIE, San Diego (2008).

[7] F. Beckmann, [Microtomography using synchrotron radiation as a user experiment at beamlines BW2 and BW5 of HASYLAB at DESY] SPIE, San Diego, USA (2002).

[8] B. Müller, R. Bernhardt, T. Weitkamp et al., "Morphology of bony tissues and implants uncovered by highresolution tomographic imaging," Int J Mat Res, 98(7), 613-621 (2007).

[9] A. C. Kak, and M. Slaney, [Principles of Computerized Tomographic Imaging] IEEE Press, New York (1988).

[10] P. Thurner, F. Beckmann, and B. Müller, "An optimization procedure for spatial and density resolution in hard Xray micro-computed tomography," Nucl. Instrum. Meth., 225(4), 599-603 (2004).

[11] B. Müller, P. Thurner, F. Beckmann et al., [Non-destructive three-dimensional evaluation of biocompatible materials by microtomography using synchrotron radiation] SPIE, San Diego, USA (2001). 
[12] M. Stampanoni, A. Groso, A. Isenegger et al., [Trends in synchrotron-based tomographic imaging: the SLS experience] SPIE, San Diego, USA (2006).

[13] O. Paris, "From diffraction to imaging: New avenues in studying hierarchical biological tissues with x-ray microbeams," Biointerphases, 3(2), FB16-FB26 (2008).

[14] P. Kraft, A. Bergamaschi, C. Broenimann et al., "Performance of single-photon-counting PILATUS detector modules," J. Synchrotron Rad., 16, 368-375 (2009).

[15] B. Müller, F. Beckmann, M. Huser et al., "Non-destructive three-dimensional evaluation of a polymer sponge by micro-tomography using synchrotron radiation ”Biomolecular Engineering, 19, $73-78$ (2002).

[16] F. Kernen, T. Waltimo, H. Deyhle et al., [Synchrotron radiation-based micro computed tomography in the assessment of dentin de- and re-mineralization] SPIE, San Diego (2008).

[17] S. Zabler, P. Cloetens, and P. Zaslansky, "Fresnel-propagated submicrometer x-ray imaging of water-immersed tooth dentin," Optics Lett., 32(20), 2987-2989 (2007).

[18] S. R. Stock, A. E. M. Vieira, A. C. B. Delbem et al., "Synchrotron microComputed Tomography of the mature bovine dentinoenamel junction,” J. Structural Biol., 161(2), 162-171 (2008).

[19] B. Müller, "Natural formation of nanostructures: from fundamentals in metal heteroepitaxy to applications in optics and biomaterials science," Surf. Rev. Lett., 8(1/2), 169-228 (2001).

[20] J. V. Barth, J. Weckesser, C. Cai et al., "Building supramolecular nanostructures at surfaces by hydrogen bonding," Angewandte Chemie - Int. Ed. , 39, 1230-1234 (2000). 